\title{
Image-guided high-dose-rate brachytherapy for prostate cancer patients with previous rectal resection and pelvic irradiation: feasibility study
}

\author{
Ewelina Gruszczynska, MGR!', Mateusz Dabkowski, PhD², Anetta Kasprowicz, MD², Anna Kulik, PhD², Michał Bijok, MGRl, \\ Adam Kowalczyk, MGRl', Katarzyna Sikorkka, MGRl', Agnieszka Zolciak-Siwinska, PhD² \\ 'Department of Medical Physics, The Maria Sklodowska-Curie Institute - Oncology Center, Warsaw, Poland, '2Department of Brachytherapy, \\ The Maria Sklodowska-Curie Institute - Oncology Center, Warsaw, Poland
}

\begin{abstract}
Purpose: The aim of this study was to describe treatment procedure and early clinical outcomes of high-dose-rate (HDR) interstitial brachytherapy (BT) in clinically localized prostate cancer patients previously treated for rectal cancer with abdominoperineal rectal resection and external beam radiation therapy (EBRT).

Material and methods: Between February and July 2015, two patients with clinically localized prostate cancer without rectal access were treated in our brachytherapy department. HDR interstitial brachytherapy was conducted with the guidance of fluoroscopy and computed tomography (CT) imaging. Brachytherapy was combined with hormonal therapy.

Results: Follow-up lasted for 34 and 39 months for patient 1 and 2, respectively. Both patients remained free from biochemical recurrence according to the Phoenix definition. No severe G3/G4 late toxicity was observed, and neither patient experienced any gastrointestinal morbidity. Acute and late urinary toxicities were at an acceptance level, and were scored G1 and G2 for patient 1, and G3 and G1 for patient 2, respectively.

Conclusions: Fluoroscopy and 3D CT image-guided interstitial brachytherapy is feasible and appears to be a suitable treatment technique for patients with clinically localized prostate cancer after previous rectal resection and external beam radiation therapy.

Key words: brachytherapy, HDR, rectal cancer, prostate cancer.

\section{Purpose}

Prostate cancer patients with a previous history of rectal cancer may cause significant clinical problems for uro-oncology team clinicians. Moreover, abdominoperineal rectal resection and pelvic external beam radiotherapy make the subsequent treatment of prostate cancer difficult. Urologists may consider these patients as inoperable, and re-irradiation with external beam radiation therapy (EBRT) might be ruled out. Also, brachytherapy (BT) as a treatment option remains very challenging, as there is no rectal access present for ultrasound guidance for a needle insertion. On the other hand, interstitial brachytherapy has a well-established place both as a definitive treatment $[1,2,3]$ and re-treatment of radio-recurrent prostate cancer $[4,5,6]$. This is the reason why the clinical team of our brachytherapy department decided to take up the challenge to perform a brachytherapy treatment for patients with no rectal access.

\section{Material and methods}

\section{Patient 1}

59-year-old male diagnosed with high-risk prostate cancer in 2014.

In 2009, the patient was diagnosed with cT3N2 rectal adenocarcinoma. He was treated with preoperative pelvic EBRT (25 Gy in 5 fractions), followed by abdominoperineal rectal resection and adjuvant chemotherapy (5-fluorouracil and leucovorine - 15 cycles). Until now, he remains free from the progression of rectal cancer.

In 2011, an elevated level of prostate-specific antigen (PSA) was detected. It gradually increased from $6.92 \mathrm{ng} /$ $\mathrm{ml}$ up to $21.63 \mathrm{ng} / \mathrm{ml}$ in 2014. The patient underwent several transperineal ultrasound (US)-guided prostate biopsies without confirmation of prostate cancer. Multiparametric magnetic resonance imaging (MRI) of the prostate, in 2014, revealed PIRADS 4 lesions in the peripheral zone of the right lobe near the apex, with no extraprostatic ex- 
tension. The histopathologic confirmation was obtained via transperineal US-guided biopsy at the $5^{\text {th }}$ attempt. Gleason score $8(4+4)$ adenocarcinoma was confirmed. Computed tomography (CT) of the thorax, abdomen, and pelvis along with bone scintigraphy were performed to exclude metastatic disease.

At first, the patient was referred to a urologist and then to a radiation oncologist and was disqualified from further definitive treatment due to previous radiation therapy and surgery in the pelvic region.

Subsequently, he was consulted at brachytherapy department in February 2015, and the decision to perform transperineal prostate brachytherapy with 3 years of hormonal treatment was taken.

From June to July 2015, three fractions of 10 Gy were delivered to the prostate, with 21 days interval between fractions [5]. The patient complained of severe side effects of the hormonal treatment; therefore, the hormonal treatment was terminated in October 2016.

\section{Patient 2}

64-year-old male diagnosed with intermediate-risk prostate cancer in 2014.

The patient was diagnosed and treated for rectal cancer in 2009. He underwent an anterior rectal resection in June 2009, which revealed a pT2N0 G2 adenocarcinoma of the rectum. The tumor recurred in March 2010 and was deemed inoperable due to infiltration of the sacral bone. The patient received radiochemotherapy, with pelvic irradiation (25 Gy in 5 fractions) and three cycles of FOLFOX4 chemotherapy. A surgical attempt of tumor resection was performed in August 2010. Due to sacral and left iliac bone infiltration, it was again considered inoperable. From October 2010 to January 2011, the patient received five cycles of xeloda. In February 2011, an abdominoperineal resection was performed, and histopathologic report revealed total pathological regression after radio- and

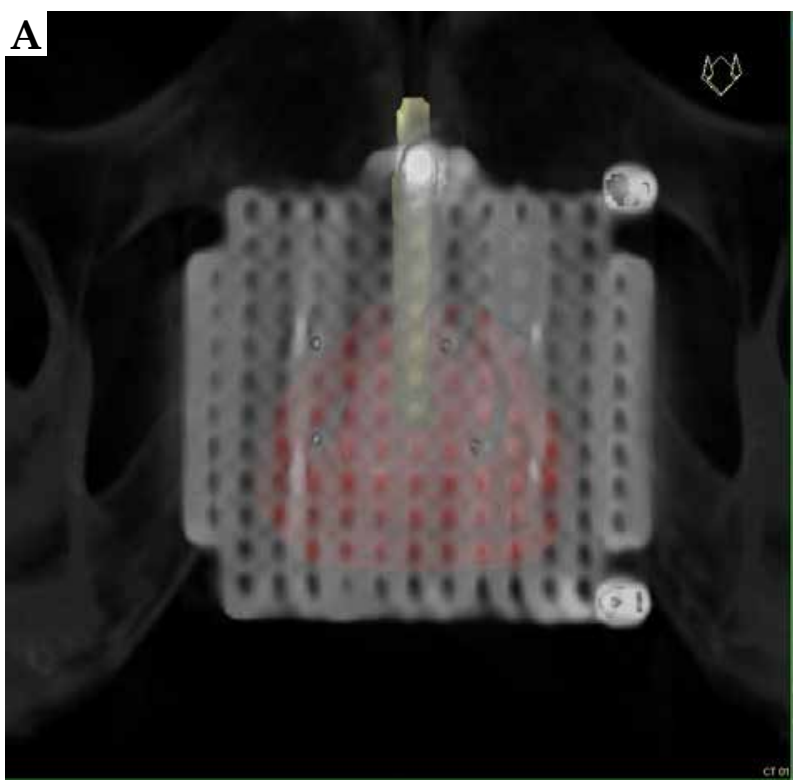

chemotherapy. Furthermore, carcinoembryonic antigen (CAE) level has been stable from the end of radical treatment of rectal cancer till now, ranging 2.8-3.5 ng/ $\mathrm{ml}$.

In 2013, the patient was referred to a urologist because of an elevated PSA level (7 ng/ml). Further follow-up examination revealed a PSA progression of up to $13.52 \mathrm{ng} /$ $\mathrm{ml}$ in 2014. The histopathologic confirmation of Gleason score $6(3+3)$ adenocarcinoma was obtained via a transperineal US-guided biopsy ( $2^{\text {nd }}$ attempt). CT of the thorax, abdomen, and pelvis as well as bone scintigraphy were performed, which excluded metastatic disease of prostate cancer and showed no residual lesions to sacral and iliac bones due to rectal cancer. In a multiparametric MRI of the prostate, two PIRADS 4 lesions were found in the right and left peripheral zone, with no extraprostatic extension.

Similarly to patient 1 , patient 2 was excluded from both radical prostatectomy and external beam radiotherapy. The patient was referred to brachytherapy department in November 2014 and from February to March 2015, he underwent an interstitial treatment. Brachytherapy consisted of three $9.5 \mathrm{~Gy}$ fractions delivered to the prostate, with 21 days interval between fractions, combined with 6 months of hormonal treatment was ordered.

\section{Application technique}

The procedure was performed under spinal anesthesia. After setting the patient in the lithotomy position, a urinary catheter was inserted into the bladder. Then, the prostate template was sutured to the patient's perineum and the patient's legs were put down for CT examination. The computed tomography, which was located in the operating room and moved on rails, was performed with scans of $2 \mathrm{~mm}$ thickness. The visible template, prostate, and urethra were contoured to obtain a $3 \mathrm{D}$ view, which allowed to determine coordinates essential for the insertion of first needles, avoiding the urethra. The insertion depth was measured on CT in the sagittal plane (Figure 1).

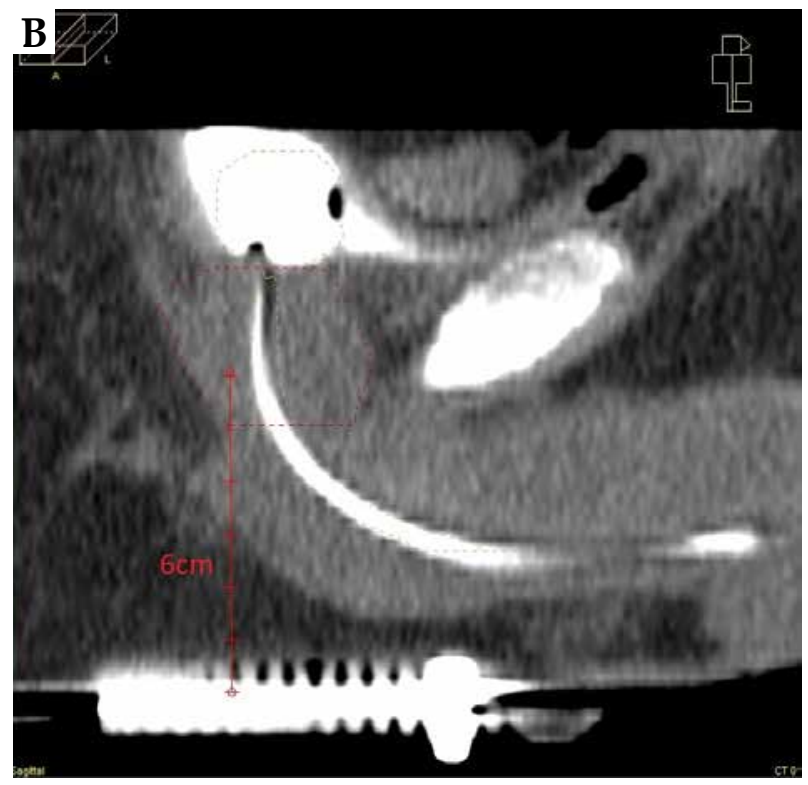

Fig. 1. 3D view of prostate and urethra with the template to choose the hole for the insertion needles (A). B) Sagittal view of the measurement of the depth, at which needle has to be inserted 

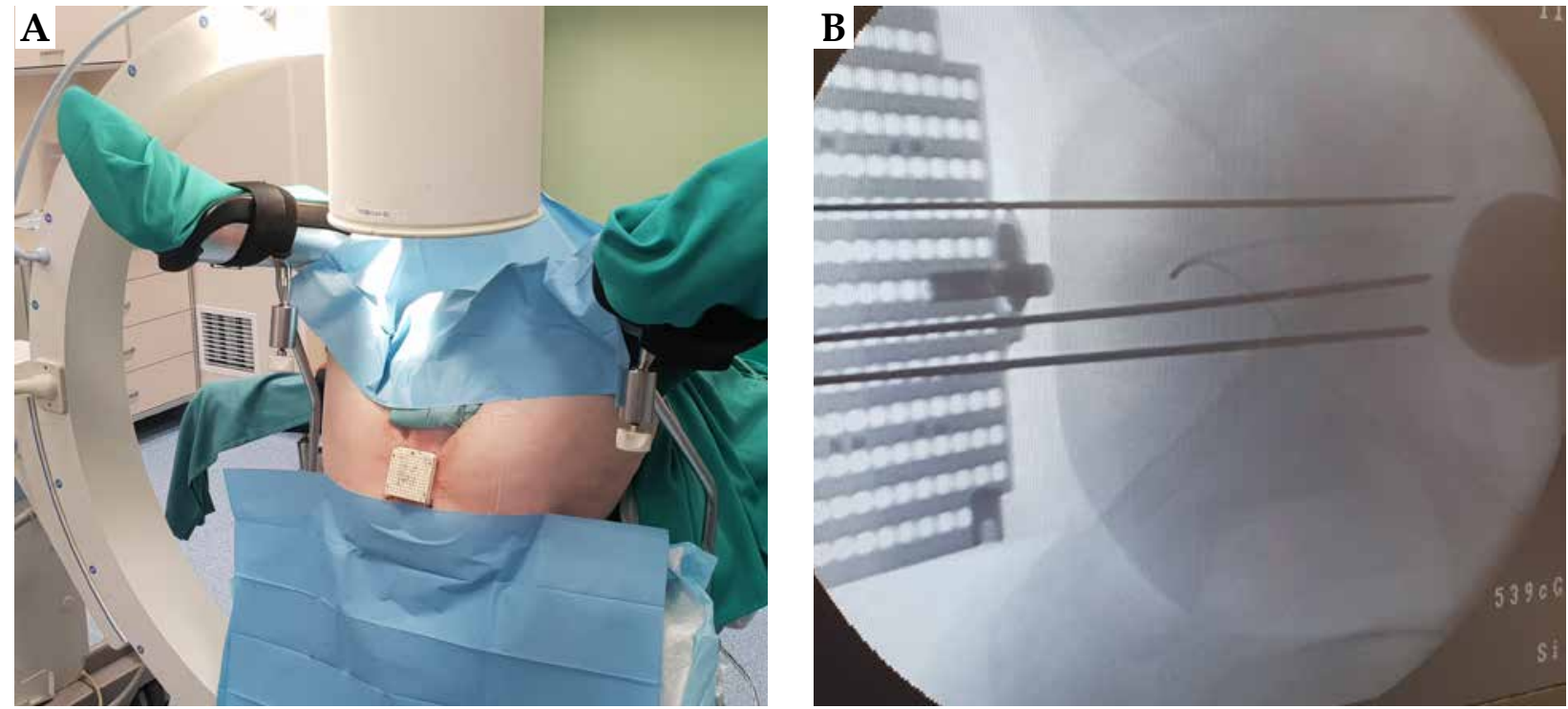

Fig. 2. The first needles inserted via prostate template under fluoroscopy guidance with a contrast agent into the bladder and Foley catheter

Flexible plastic needles with stylets (Elekta AB, Stockholm, Sweden) were used for the implant procedure. The insertion of first needles was performed under the guidance of fluoroscopy (Figure 2). For better recognition of the patient's anatomy, a contrast agent was injected into the bladder and the Foley balloon catheter. After insertion of the first four needles, the stylets were changed to CT markers and a CT scan was performed. Afterwards, a virtual simulation procedure with prostate delineation and template reconstruction permitted for planning the coordinates and depth of further needles. All the needles were inserted under the guidance of fluoroscopy. After implantation was completed, a final CT imaging with $2 \mathrm{~mm}$ slice thickness was undertaken for 3 dimensional (3D) conformal planning, using Oncentra Brachy version 4.3 planning system (Elekta AB, Stockholm, Sweden). The planning target volume (PTV), urethra, intestine, and bladder were contoured, and the PTV consisted of prostate gland without any margins. Treatment delivery was performed on microSelectron HDR (Elekta $A B$, Stockholm, Sweden) in supine position, similar to that during CT. Treatment plan acceptance depended on the fulfillment of PTV coverage requirements in compliance with organ at risk constraints (Table 1).

After irradiation and removal of the needles, an ink tattoo was made on the perineum to facilitate the positioning of template and the insertion of needles during the second and third fraction (Figure 3). The whole pro- cedure with fluoroscopy guidance and CT virtual simulation was repeated for all fractions.

Toxicity was reported according to the Common Toxicity Criteria of Adverse Events (CTCAE) version 4. A clinical outcome was monitored using PSA measurements. The follow-up visits were scheduled every 3 months for 2 years and every 6 months for the next 3 years.

\section{Results}

The follow-up time was 34 and 39 months for patient 1 and 2, respectively. Table 2 shows the prostate, urethral, bladder, and intestines dosimetry parameters achieved. Since it was a re-irradiation, the analysis of cumulative doses in organs at risk (OARs) was necessary. The doses were converted to equivalent dose of 2 Gy fractions $\left(\mathrm{EQD}_{2}\right)$, according to the linear-quadratic model, and adopting an $\alpha / \beta$ value of 3 for organs at risk and an $\alpha / \beta$ value of 1.5 for the prostate $[7,8,9]$. The dose distributions for both patients are presented on Figure 4.

For the first patient, the $\mathrm{D}_{90}$ for PTV was lower than the prescribed dose because of high-dose to the urethra. The intestines in patient 1 were situated above the bladder, in contrast to patient 2, where one loop of intestines was located below the bladder between the prostate and sacral bone.

Acute toxicity was restricted to the genitourinary tract, with no complaints regarding the gastrointestinal

Table 1. Total physical prescription doses for planning target volume (PTV) and normal tissue dose constraints (as a percentage of prescribed dose or $\mathrm{EQD}_{2}$ dose value)

\begin{tabular}{lcccc} 
& PTV & Urethra & $\begin{array}{c}\text { Bladder } \\
D_{\text {max }} / D_{0.1 m^{3}}\end{array}$ & $D_{\max }$ \\
\hline$D_{90}$ & $V_{100}$ & $V_{200}$ & $<120 \% /<\mathrm{EQD}_{2}=120$ Gy & $<85 \%$
\end{tabular}

$D_{90}$-minimum dose to hottest $90 \%$ of the volume, $V_{100}, V_{200}$-volume receiving at least $100 \%, 200 \%$ of the prescribed dose, $D_{\text {max }}-$ maximum dose in the volume, $D_{0.1 \mathrm{~cm}^{3}}$ - minimum dose to hottest $0.1 \mathrm{~cm}^{3}$ volume, $E Q D_{2}$ - equivalent dose in 2 Gy fractions 

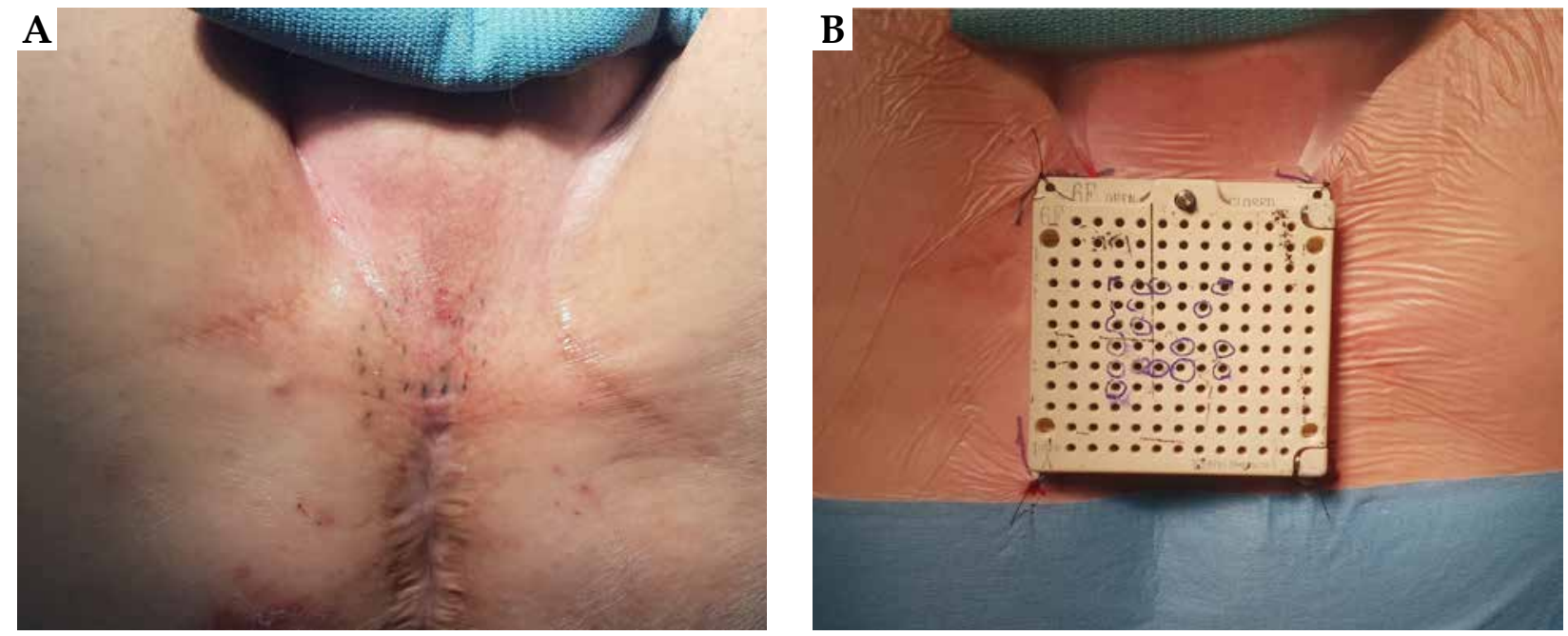

Fig. 3. Ink tattoo on perineum (A). Template fixed to the patient's perineum with tattoo guidance (B)

tract. In patient 1, G1 was scored with transient hematuria, dysuria, and mild urinary frequency. Patient 2 suffered from acute urinary retention after third fraction (G3 acute toxicity). The Foley catheter was removed 2 months after insertion. Late toxicity was low, we did not notice any severe (G3/G4) GI and GU side effects. The patients complained of mild urinary flow restriction, which was resolved with tamsulosine. The immobilization and implantation procedure were well tolerated by the patients.

Patients 1 and 2 remained free from biochemical recurrence according the Phoenix definition up to the last follow-up visit. Patient 1 achieved PSA nadir in June 2015 $(<0.003 \mathrm{ng} / \mathrm{ml})$ and his actual PSA level is $0.007 \mathrm{ng} / \mathrm{ml}$. The second patient achieved PSA nadir of $0.6 \mathrm{ng} / \mathrm{ml}$ in November 2016 and the PSA level at last follow-up visit was $1.91 \mathrm{ng} / \mathrm{ml}$.

\section{Discussion}

There is a limited treatment option for a newly diagnosed prostate cancer in patients with a previous history of pelvic surgery and pelvic irradiation due to rectal cancer. An active surveillance is indicated for low-risk prostate cancer, and since our patients were the intermediateand high-risk group patients, it was not an option. The patients were also disqualified from radical prostatectomy due to expected technical difficulties associated with this procedure and a high probability of urinary tract complications. Moreover, EBRT was not possible due to re-irradiation settings in the pelvic area.

Despite many difficulties, including technical access, we obtained a similar dose distribution for PTV and OARs as the patients treated routinely in our department, using real-time transrectal ultrasound (TRUS)-based image guidance techniques. The doses delivered for targets were in accordance with the recommendations and the doses for OARs were in the range of tolerated doses [9]. The limitation of our study is the uncertainty of the calculated doses using linear-quadratic (LQ) model. This model is reasonably well validated, experimentally and theoretically, up to about 10 Gy per fraction [10]. The second
Table 2. The doses and cumulative doses given to planning target volume (PTV) and organs at risk

\begin{tabular}{|c|c|c|}
\hline Parameter & Patient 1 & Patient 2 \\
\hline Age (year) & 59 & 65 \\
\hline Previous pelvic EBRT dose (Gy) & 25 & 25 \\
\hline Prescribe HDR dose (Gy) & 30 & 28.5 \\
\hline $\begin{array}{l}\text { PTV prescribed } \\
\mathrm{EQD}_{2} \alpha / \beta=1.5 \text { (Gy) }\end{array}$ & 98.6 & 89.6 \\
\hline PTV volume $\left(\mathrm{cm}^{3}\right)$ & 31.5 & 35.5 \\
\hline $\mathrm{D}_{90}(\%)$ & 99.2 & 100.3 \\
\hline $\mathrm{V}_{100}(\%)$ & 89.5 & 90.3 \\
\hline $\mathrm{V}_{150}(\%)$ & 37.0 & 33.1 \\
\hline$V_{200}(\%)$ & 13.3 & 15.5 \\
\hline $\mathrm{EQD}_{2} \alpha / \beta=1.5 \mathrm{D}_{90}(\mathrm{~Gy})$ & 97 & 90.2 \\
\hline \multicolumn{3}{|l|}{ Urethra } \\
\hline $\mathrm{D}_{\max }(\%)$ & 124.0 & 119.3 \\
\hline $\mathrm{D}_{0.1 \mathrm{~cm}^{3}}(\%)$ & 119.2 & 116.0 \\
\hline BT EQD $2 D_{0.1 \mathrm{~cm}^{3}}(\mathrm{~Gy})$ & 106.7 & 92.7 \\
\hline $\mathrm{D}_{10}(\%)$ & 118.4 & 114.7 \\
\hline $\mathrm{D}_{30}(\%)$ & 112.8 & 110,3 \\
\hline CumEQD $_{2} D_{0.1 \mathrm{~cm}^{3}}$ (Gy) & 146.7 & 132.7 \\
\hline \multicolumn{3}{|l|}{ Bladder } \\
\hline $\mathrm{D}_{\max }(\%)$ & 84.8 & 87.9 \\
\hline $\mathrm{D}_{2 \mathrm{~cm}}(\%)$ & 53.2 & 59.1 \\
\hline CumEQD $_{2} \mathrm{D}_{2 \mathrm{~cm}^{3}}$ (Gy) & 66.8 & 69.2 \\
\hline \multicolumn{3}{|l|}{ Intestines } \\
\hline $\mathrm{D}_{\max }(\%)$ & 13.2 & 62.6 \\
\hline CumEQD $2 D_{2 \mathrm{~cm}^{3}}$ (Gy) & 42.4 & 50.8 \\
\hline
\end{tabular}

$D_{90}$ - minimum dose to hottest $90 \%$ volume, $V_{100}, V_{150}, V_{200}$-volume receiving at least $100 \%, 150 \%, 200 \%$ of prescribed dose, $D_{\max }$ - maximum dose of the organ, $D_{0.1 \mathrm{~cm}^{3}}, D_{2 \mathrm{~cm}^{3}}$ - minimum dose to hottest $0.1 \mathrm{~cm}^{3}$ and $2 \mathrm{~cm}^{3}$ volume, $D_{10}$ $D_{30}$ - minimum dose to hottest $10 \%, 30 \%$ volume, BT EQD 2 - equivalent dose in 2 Gy fractions for brachytherapy, CUmEQD - cumulative equivalent dose in 2 Gy fractions for brachytherapy and external beam radiation therapy 

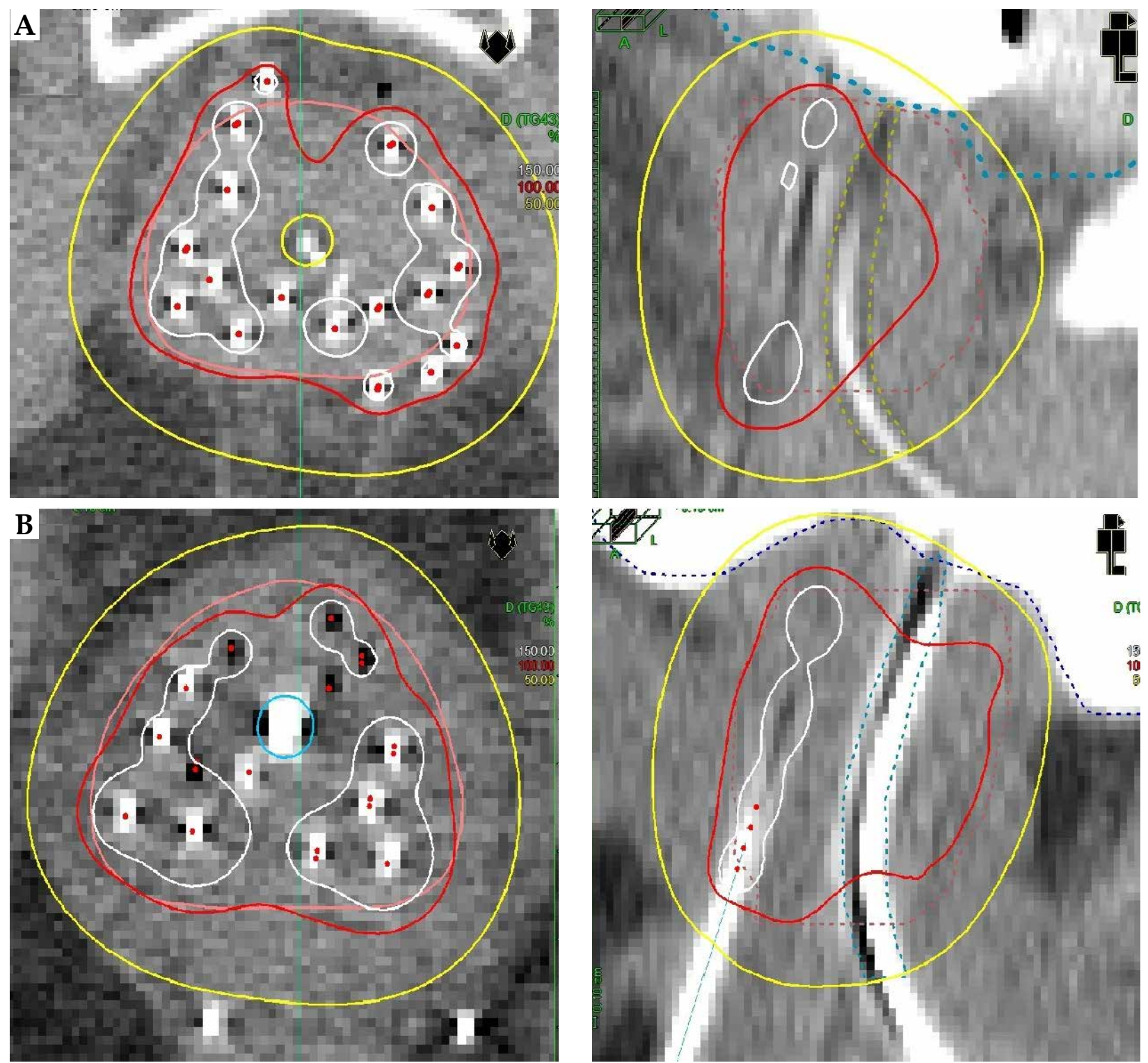

Fig. 4. Dose distribution for patient 1 (A) and patient 2 (B) (transverse view - left, sagittal view - right). The code for the isodose color is as follows: white $150 \%$, red $100 \%$, yellow $50 \%$

limitation is that we were unable to performed plan fusion from external beam radiation therapy and brachytherapy treatments. We adopted the worst-case scenario; the most exposed parts of OARs to BT dose received the total prescribed dose from EBRT. The total cumulative $\mathrm{EQD}_{2}$ for patient 1 and patient 2 for the bladder were 64.8 and 68.1 Gy, for the urethra 146.4 and $132.4 \mathrm{~Gy}$, and for the intestines 42.4 and $50.8 \mathrm{~Gy}$, respectively. Our results of the cumulative doses for the bladder and intestines are lower than reported in the literature for re-irradiation in the pelvic area. Zolciak-Siwinska et al. [11] treated 20 patients with re-irradiation of cervical or vaginal cancer within a previously irradiated area. A cumulative $\mathrm{EQD}_{2}$ of $2 \mathrm{~cm}^{3}$ of the bladder was $99.3 \mathrm{~Gy}$. Similar results were reported by Abusaris et al. [12] who treated 23 patients after a third radiation treatment, with a partial or complete overlap of the previous two irradiated regions.
The median corrected cumulative dose for the intestines and bladder resulted in 73 Gy and 79 Gy, respectively, and was well tolerated by the patients.

To our knowledge, there are only two reports in the literature describing treatment of prostate cancer with brachytherapy in this difficult clinical scenario. Jabbari et al. [13] presented results for four patients and Koutrouvelis et al. [14] for six. Jabbari described a very interesting and elegant technique, where the first set of needles was implanted in the prostate under transperineal US guidance. Furthermore, the depth of the needles inserted was checked via cystoscopy, which confirmed tip tenting of the bladder mucosa. The subsequent needles were inserted with the help of a transperineal silicone template. He used a salvage fractionation regimen of 36 Gy in 6 fractions and two implants. In order to minimize the intrafraction movement, the catheters were fixed to the 
perineum using either friction buttons, silicon template, or a dental hardened polymer sutured to perineal skin. Our team tried to use the transperineal US as described above, but we did not manage to obtain images clear enough to begin the implantation.

Koutrouvelis performed 125-Iodine LDR brachytherapy under CT guidance only, but with the use of more sophisticated 3D stereotactic navigation system to guide the insertion of catheters. However, this procedure requires multiple CT scans to verify the correct needle position for every transverse row of needles before the implantation. If a stereotactic 3D navigation system is available, the procedure described by our team seems to be feasible and reproducible. We inserted the first 4 needles under the guidance of fluoroscopy and then, a CT scan was performed. Afterwards, a virtual simulation procedure with prostate delineation and template reconstruction allowed for planning the coordinates and the depth of further needles.

\section{Conclusions}

Fluoroscopy and 3D CT image-guided interstitial brachytherapy is feasible and appears to be an adequate method of treatment for patients with clinically localized prostate cancer after previous rectal resection and external beam radiation therapy.

\section{Disclosure}

Authors report no conflict of interest.

\section{References}

1. Tselis N, Hoskin P, Baltas D et al. High dose rate brachytherapy as monotherapy for localised prostate cancer: review of the current status. Clin Oncol 2017; 29: 401-411.

2. Strouthos I, Tselis N, Chatzikonstantinou G et al. High dose rate brachytherapy as monotherapy for localised prostate cancer. Radiother Oncol 2018; 126: 270-277.

3. Kukielka A, Dabrowski T, Walasek T et al. High-dose-rate brachytherapy as a monotherapy for prostate cancer. Single-institution results of the extreme fractionation regimen. Brachytherapy 2015; 14: 359-365.

4. Chatzikonstantinou G, Zamboglou N, Rödel C et al. Highdose-rate brachytherapy as salvage modality for locally recurrent prostate cancer after definitive radiotherapy: a systematic review. Strahlenther Onkol 2017; 193: 683-691.

5. Wojcieszek P, Szlag M, Glowacki G et al. Salvage high-doserate brachytherapy for locally recurrent prostate cancer after primary radiotherapy failure. Radiother Oncol 2016; 119: 405410.

6. Parekh A, Graham P, Nguyen P. Cancer control and complications of salvage local therapy after failure of radiotherapy for prostate cancer: a systematic review. Semin Radiat Oncol 2013; 23: 222-234.

7. Fowler JF, Toma-Dasu I, Dasu A. Is the $\alpha / \beta$ ratio for prostate tumours really low and does it vary with the level of risk at diagnosis? Anticancer Res 2013; 33: 1009-1011.

8. Vogelius IR, Bentzen SM. Dose response and fractionation sensitivity of prostate cancer after external beam radiation therapy: a meta-analysis of randomized trials. Int J Radiat Oncol Biol Phys 2018; 100: 858-865.
9. Hoskin P, Colombo A, Henry A et al. GEC/ESTRO recommendations on high dose rate afterloading brachytherapy for localized prostate cancer: An update. Radiother Oncol 2013; 107: 325-332.

10. Brenner DJ. The linear-quadratic model is a appropriate methodology for determining isoeffective doses at large doses per fraction. Semin Radiat Oncol 2008; 18: 234-239.

11. Zolciak-Siwinska A, Bijok M, Jonska-Gmyrek J et al. HDR brachytherapy for the reirradiation of cervical and vaginal cancer: analysis of efficacy and dosage delivered to organs at risk. Gynecol Oncol 2014; 132: 93-97.

12. Abusaris H, Storchi PRM, Brandwijk RP et al. Second re-irradiation: efficacy, dose and toxicity in patients who received three courses of radiotherapy with overlapping fields. Radiother Oncol 2011; 99: 235-239.

13. Jabbari S, Hsu IC, Kawakami J et al. High-dose-rate brachytherapy for localized prostate adenocarcinoma post abdominoperineal resection of the rectum and pelvic irradiation: Technique and experience. Brachytherapy 2009; 8: 339344.

14. Koutrouvelis P, Theodorescu D, Katz S et al. Brachytherapy of prostate cancer after colectomy for colorectal cancer: pilot experience. J Urol 2005; 173: 82-86. 